\title{
Direct evidence for the onset of intruder configurations in neutron-rich $\mathrm{Ne}$ isotopes
}

\author{
J.R. Terry ${ }^{\mathrm{a}, \mathrm{b}, *}$ D. Bazin ${ }^{\mathrm{b}}$ B.A. Brown ${ }^{\mathrm{a}, \mathrm{b}}$ C.M. Campbell ${ }^{\mathrm{a}, \mathrm{b}}$ \\ J.A. Church ${ }^{a, b, 1}$ J.M. Cook ${ }^{a, b}$ A.D. Davies ${ }^{a, b}$ D.-C. Dinca ${ }^{a, b, 2}$ \\ J. Enders ${ }^{b, 3}$ A. Gade ${ }^{b}$ T. Glasmacher ${ }^{a, b}$ P.G. Hansen ${ }^{a, b}$ \\ J.L. Lecouey ${ }^{b}$ T. Otsuka ${ }^{\text {d,e }}$ B. Pritychenko ${ }^{g}$ B.M. Sherrill ${ }^{a, b}$ \\ J.A. Tostevin ${ }^{c}$ Y. Utsuno ${ }^{f}$ K. Yoneda ${ }^{b, 4}$ H. Zwahlen ${ }^{a, b}$ \\ ${ }^{a}$ Department of Physics and Astronomy, Michigan State University, East Lansing, \\ MI 48824, USA \\ ${ }^{\mathrm{b}}$ National Superconducting Cyclotron Laboratory, Michigan State University, East \\ Lansing, MI 48824, USA \\ ${ }^{\mathrm{c}}$ School of Electronics and Physical Sciences, University of Surrey, Guildford GU2 \\ $7 X H, U K$ \\ ${ }^{\mathrm{d}}$ Department of Physics and Center for Nuclear Study, University of Tokyo, \\ Hongo, Tokyo 113-0033, Japan \\ e RIKEN, Hirosawa, Wako-shi, Saitama 351-0198, Japan \\ ${ }^{\mathrm{f}}$ Japan Atomic Energy Research Institute, Tokai, Ibaraki 319-1195, Japan \\ ' Brookhaven National Laboratory, Upton, NY 11973-5000 USA
}

\begin{abstract}
Monte Carlo shell-model calculations with the modern SDPF-M interaction successfully describe neutron-rich nuclei in the vicinity of $N=20$ where normal and intruder configurations coexist at low excitation energy. We report on direct experimental evidence of the population of the $3 / 2^{-}$intruder state in ${ }^{27} \mathrm{Ne}$ in the knockout of a single neutron from the ground state of ${ }^{28} \mathrm{Ne}$. This low-lying negative parity state is consistent with a narrower shell gap for exotic nuclei with $Z \ll N$ and $N \approx 20$. This observation also demonstrates the importance of direct reactions for the study of exotic nuclei and the predictive power of these large-scale shell-model calculations.
\end{abstract}

Key words: intruder states, knockout, ${ }^{25} \mathrm{Ne},{ }^{26} \mathrm{Ne},{ }^{27} \mathrm{Ne},{ }^{28} \mathrm{Ne}$ PACS: 27.30.+t, 21.10.Jx, 21.60.Cs, 25.60.Gc 
Neutron-rich nuclei with atomic numbers between $Z \approx 8$ and $Z \approx 12$ exhibit dramatic differences in their structure compared to stable isotopes, already at the level of shell closures [1]. While ${ }^{16} \mathrm{O}$ is a prototypical doubly-magic nucleus with a shell gap of $10 \mathrm{MeV}$ [2], the ground state of ${ }^{28} \mathrm{O}$, with canonical magic numbers $Z=8$ and $N=20$, is not particle-bound: The $N=20$ shell closure is not strong enough to bind ${ }^{28} \mathrm{O}$. Contrary to expectations, recent experiments have indicated a pronounced $N=16$ subshell closure [3-5] culminating in the identification of ${ }^{24} \mathrm{O}$ as a doubly magic nucleus [4] and leading to the revision of the effective interaction for the $s d$-shell $[1,6]$. Adding one proton to the heaviest particle-bound oxygen isotope $\left({ }^{24} \mathrm{O}\right)$ dramatically shifts the neutron dripline at $Z=9$ and allows for the binding of at least six additional neutrons [7]. The $N=20$ major shell closure also breaks down for ${ }^{31} \mathrm{Na}$ and ${ }^{32} \mathrm{Mg}$ as indicated by an excess of binding energy $[8,9]$ and by measurements sensitive to collectivity [10-12]. Both observations, supported by theory, indicate a region of $N \geq 20$ and $Z \leq 12$, referred to as the "island of inversion" [13], in which the ground-state wave function is dominated by intruder configurations characterized by particle-hole excitations across the $N=20$ shell gap.

These observations have posed a formidable challenge to nuclear structure models. The recently-developed Monte Carlo shell model (MCSM) [14] with the new SDPF-M interaction [6], referred to as SDPF-M calculations, has had great success in accurately reproducing structure changes in this region. This theoretical approach allows unrestricted mixing of particle-hole configurations across the $N=20$ shell gap. For ${ }^{29} \mathrm{~F}$, a considerable $4 \mathrm{p}-4 \mathrm{~h}$ component in the ground state in addition to enhanced pairing leads to a bound ground state, explaining the puzzling extension of the dripline at $Z=9$ [15]. Conventional shell-model calculations within a configuration space limited to the $s d$-shell with the Universal SD (USD) interaction [16], referred to as USD calculations, have been shown to have high predictive power. However, calculations with the USD interaction do not allow for intruder configurations across the $N=$ 20 gap, and thus they fail to reproduce shell-breaking effects near $N=20$. Between the enhanced $N=16$ sub-shell closure and the reduced $N=20$ shell gap, SDPF-M predictions compare well with known level structures and electromagnetic moments of ${ }^{27-31} \mathrm{Na}$, illustrating the power of a consistent approach in this transitional region $[1,17,18]$.

USD and SDPF-M calculations make very different predictions for the neutron-

\footnotetext{
* terry@nscl.msu.edu

1 Present address: Lawrence Livermore National Laboratory, Livermore, California 94550 USA

2 Present address: American Science \& Engineering, Inc., Billerica, Massachusetts 01821 USA

3 Present address: Institut für Kernphysik, Technische Universität Darmstadt, D64289 Darmstadt, Germany

4 Present address: RIKEN, Hirosawa, Wako-shi, Saitama 351-0198, Japan
} 
rich Ne isotopes, which are located in a transitional region between $N=16$ and $N=20$. SDPF-M calculations indicate that intruder configurations are important already for the $N=17^{27} \mathrm{Ne}$, even in the low-lying level scheme. The same calculations predict that intruder states in ${ }^{25,26} \mathrm{Ne}$ lie above $3 \mathrm{MeV}$. However, with just one additional neutron, the first intruder state in ${ }^{27} \mathrm{Ne}$ is expected to be nearly degenerate with the non-intruder ground state. Fig. 1 illustrates this dramatic change in structure for the Ne isotopes. For ${ }^{25} \mathrm{Ne}$ the USD-calculated level scheme and the predictions from the SDPF-M calculation agree, while for ${ }^{27} \mathrm{Ne}$ two additional states are expected below the excited $1 / 2^{+}$state. These two levels are negative-parity intruder states originating from particle-hole excitations across the $N=20$ shell gap.

A recent systematic investigation of the level structures of ${ }^{25-29} \mathrm{Ne}$ by in-beam $\gamma$-ray spectroscopy following fragmentation indicates - contrary to SDPF-M predictions - that ${ }^{27} \mathrm{Ne}$ can be well described without considering intruder effects and that the onset of intruder admixture occurs at the $N=18^{28} \mathrm{Ne}$ [19]. In contrast, a recent $(d, p)$ measurement populating states in ${ }^{27} \mathrm{Ne}$ reveals two excited states at low excitation energy, which models without cross-shell excitations cannot reproduce [20].

In the present work, the two key nuclei ${ }^{25} \mathrm{Ne}$ and ${ }^{27} \mathrm{Ne}$ are investigated using the direct ${ }^{9} \mathrm{Be}\left({ }^{26} \mathrm{Ne},{ }^{25} \mathrm{Ne}\right) \mathrm{X}$ and ${ }^{9} \mathrm{Be}\left({ }^{28} \mathrm{Ne},{ }^{27} \mathrm{Ne}\right) \mathrm{X}$ one-neutron knockout reactions, in order to firmly establish the role of intruder configurations in these nuclei. Aside from $\gamma$-ray transitions yielding the energies of excited states, information on the details of the nuclear wave functions are accessible from single-nucleon knockout reactions [21]. The shape of the longitudinal momentum distributions of the knockout residues depends on the $\ell$-value of the knocked-out neutron and cross sections to individual excited states can be measured. From these, in comparison to reaction theory, spectroscopic factors can be derived, which relate to the occupation numbers of single-particle orbits. Population of intruder states in ${ }^{27} \mathrm{Ne}$ in the knockout from ${ }^{28} \mathrm{Ne}$ would provide direct evidence for intruder components in the ground-state wave function of ${ }^{28} \mathrm{Ne}$.

The experiments were performed at the Coupled-Cyclotron Facility [22] of the National Superconducting Cyclotron Laboratory at Michigan State University. The ${ }^{26} \mathrm{Ne}$ and ${ }^{28} \mathrm{Ne}$ secondary beams were produced by fragmentation of $140 \mathrm{MeV} /$ nucleon primary beams of ${ }^{40} \mathrm{Ar}$ and ${ }^{48} \mathrm{Ca}$ on 587 and $846 \mathrm{mg} / \mathrm{cm}^{2}$ beryllium targets, respectively. The fragments were magnetically analyzed in the A1900 fragment separator [23]. In both cases, the secondary beam was impinged on a $376 \mathrm{mg} / \mathrm{cm}^{2}$ beryllium target placed at the target position of the S800 spectrograph [24] at mid-target energies of 83 and $80 \mathrm{MeV} /$ nucleon, respectively. The reaction residues were identified event-by-event with the focalplane detector system of the spectrograph. The position information provided by the position-sensitive detectors yielded the longitudinal momentum distribution of the reaction residues. The Segmented Germanium Array [25] sur- 
rounded the target for detection of coincident $\gamma$-ray radiation. For the ${ }^{26} \mathrm{Ne}$ experiment, the array was arranged in a two-ring configuration as in [26], while, for ${ }^{28} \mathrm{Ne}$, it was configured in nine angle pairs ranging from $24^{\circ}$ to $147^{\circ}$.

The Doppler-reconstructed $\gamma$-ray spectra in coincidence with the ${ }^{27} \mathrm{Ne}$ and ${ }^{25} \mathrm{Ne}$ knockout residues, respectively, are shown in Fig. 2. The $\gamma$-ray spectrum originating from ${ }^{25} \mathrm{Ne}$ is shown in the upper panel. The doublet observed at about $1.7 \mathrm{MeV}$ and the $2.09 \mathrm{MeV}$ transition are in good agreement with the data established from $\beta$ decay $[27,28]$. In ${ }^{27} \mathrm{Ne}$ (lower panel), three $\gamma$-rays are observed at $0.119,0.765$, and $0.885 \mathrm{MeV}$. The 0.119 and $0.765 \mathrm{MeV} \gamma$-rays are found to be in coincidence suggesting the population of only two excited states as shown in the inset of Fig. 2. The ordering of the 0.119 and 0.765 $\mathrm{MeV}$ transitions is determined from intensity balancing. The $0.119 \mathrm{MeV} \gamma-$ ray transition has not been reported in previous experiments due to higher energy thresholds. Based on the symmetric shape of the $0.885-\mathrm{MeV}$ photopeak, an upper limit of $10 \mathrm{ps}$ is placed on the halflife of the $0.885-\mathrm{MeV}$ state. The measured $19.3(25) \%$ branching ratio of the $0.119-\mathrm{MeV}$ transition relative to the $0.885-\mathrm{MeV}$ intensity, along with the upper limit on the halflife, limits the possible multipolarities to $E 1$ or $M 1$ for the $0.119-\mathrm{MeV}$ transition and $E 2$, $E 1$, or $M 1$ for the $0.885-\mathrm{MeV}$ transition.

The proposed level schemes for ${ }^{25} \mathrm{Ne}$ and ${ }^{27} \mathrm{Ne}$ are shown as insets in Fig. 2. The level scheme for ${ }^{25} \mathrm{Ne}$ is in agreement with both the USD and SDPF-M shell model predictions. For ${ }^{27} \mathrm{Ne}$ two excited states are firmly established in the present experiment. This result is in agreement with [5] and contradicts the observations and conclusions of [19]. The observations are in contradiction to the USD shell model, which predicts only one bound excited state, and are consistent with the SDPF-M shell model calculations as we will outline below.

In the present experiment, longitudinal momentum distributions were measured in the S800 spectrograph and provide direct experimental evidence that allows the characterization of the observed excited states. Individual momentum distributions for the knockout to specific final states of ${ }^{25} \mathrm{Ne}$ and ${ }^{27} \mathrm{Ne}$ residues, shown in Fig 3, are extracted by gating on the $\gamma$-ray spectrum (see $[29,26])$. Each distribution is overlaid with theoretical shapes assuming several angular momentum $\ell$-values for the knocked-out nucleons. Calculations are performed using a three-body reaction model based on the eikonal approximation [30-32]. The experimental momentum distributions are corrected for the acceptance of the S800 spectrograph.

For ${ }^{25} \mathrm{Ne}$, the distribution in coincidence with the $1.7-\mathrm{MeV}$ doublet, shown in Fig. 3, includes contributions from both the 1.7 and $3.3 \mathrm{MeV}$ levels. The excited-state distribution is in good agreement with an assignment of $\ell=2$ and not $\ell=0$. Although statistics do not allow for a similar analysis of events populating the $2.09 \mathrm{MeV}$ state, recent $\beta$-decay [28] and $d\left({ }^{24} \mathrm{Ne},{ }^{25} \mathrm{Ne}\right) p$ results [33] 
indicate spin and parity $3 / 2^{+}$for this state. The ground-state distribution is shown in the top left panel of Fig. 3 in good agreement with $\ell=0$ allowing for the unambiguous spin-parity assignment of $1 / 2^{+}$for the ground state of ${ }^{25} \mathrm{Ne}$. The results are in agreement with the USD and SDPF-M calculations, directly supporting the spin assignments given in the level schemes inset in Fig. 2 .

For ${ }^{27} \mathrm{Ne}$, the distributions extracted for the $0.765 \mathrm{MeV}$ and $0.885 \mathrm{MeV}$ photopeaks are both clearly more narrow than a calculated distribution assuming $\ell=2$, which has a width of $255 \mathrm{MeV} / \mathrm{c}$ (FWHM) after converting to the laboratory frame and folding in the momentum resolution. For the distribution corresponding to the $0.885 \mathrm{MeV}$ transition, which represents events directly feeding this excited state, a definitive assignment of $\ell=0$ or $\ell=1$ is difficult due to large error bars and the possibility of a low-momentum tail, observed in previous knockout measurements [26]. The $\ell=0$ and $\ell=1$ distributions superimposed on the $0.885 \mathrm{MeV}$ distribution in the upper-right panel of figure 3 represent least-squares fits over a range from 10.35 to $10.58 \mathrm{GeV} / \mathrm{c}$. Based on this analysis, neither $\ell=0$ nor $\ell=1$ can be excluded for this state. Since the $0.765 \mathrm{MeV}$ photopeak is indirectly feed from the $0.885 \mathrm{MeV}$ state, the distribution corresponding to the $0.765 \mathrm{MeV}$ transition, shown in the centerright panel of figure 3 , includes contributions from both excited states, and again $\ell=0$ and $\ell=1$ cannot be distinguished. The $0.765 \mathrm{MeV}$ distribution is overlaid with a combination of $\ell=0$ and $\ell=1$ to show consistency with tentative spin and parity assignments discussed below. The ground-state momentum distribution for ${ }^{27} \mathrm{Ne}$ is clearly broader than that of the excited states. Predictions of its shape based on the $\ell$-value content taken from USDand SPDF-M calculations for the ground state are overlaid and demonstrate consistency. From the experimental level scheme and the analysis of the momentum distributions, the two observed levels are consistent with spin and parity assignments $1 / 2^{+}$and $3 / 2^{-}$as suggested by the SDPF-M calculation (Fig. 1).

The SDPF-M calculations also suggest a $7 / 2^{-}$state at $0.22 \mathrm{MeV}$. Photon decay from this state would proceed by emission of $M 2 \gamma$ radiation with a long lifetime. The effect of the lifetime on the photopeak energy resolution in this case leads to a total loss of sensitivity to the transition in the current experiment. Gamma-rays from such long-lived states would be emitted far from the reaction target and the $\gamma$-ray detector array. Events in which an unidentified excited state is populated but not tagged are consequently misidentified as populating the ground state. Therefore, according to SDPF-M calculations, the measured ground-state cross section and momentum distribution should include both $3 / 2^{+}$and $7 / 2^{-}$contributions. The extracted ground-state distribution, with calculated curves reflecting SDPF-M- and USD-predicted $\ell$-value content overlayed, are shown in the upper right panel of Fig. 3. Within the experimental uncertainty the data are consistent with either prediction and 
therefore do not provide evidence for the existence of the $7 / 2^{-}$intruder state in ${ }^{27} \mathrm{Ne}$.

Further characterization of the observed states is obtained from the spectroscopic strengths deduced from the reaction. The inclusive cross sections for the ${ }^{9} \mathrm{Be}\left({ }^{28} \mathrm{Ne},{ }^{27} \mathrm{Ne}\right) \mathrm{X}$ and ${ }^{9} \mathrm{Be}\left({ }^{26} \mathrm{Ne},{ }^{25} \mathrm{Ne}\right) \mathrm{X}$ reactions of $66(3) \mathrm{mb}$ and $98(5) \mathrm{mb}$, respectively, are decomposed into partial cross section for the population of individual final states from the $\gamma$-ray intensities in the observed level schemes. The partial cross sections have been corrected for the finite acceptance of the S800 spectrograph.

Single-particle cross sections are calculated in the three-body reaction model developed in Refs. $[31,32]$. The eikonal core-target $S$-matrices are derived using SKX Skyrme Hartree-Fock [34] densities for the ${ }^{25,27}$ Ne residues. The $r m s$ radii of the neutron single-particle orbitals were also constrained using Hartree-Fock calculations [35]. The deduced spectroscopic factors are taken as the ratio of the measured partial cross section to the calculated single-particle cross section. The experimental results are summarized and compared to the two shell-model calculations in Table 1.

Although theoretical spectroscopic factors are not yet available from the SDPFM model, an upper limit is given by the calculated orbital occupancies $\langle\mathrm{n}\rangle_{\mathrm{SDPF}-\mathrm{M}}$, shown in Table 1. Since spectroscopic strength is often concentrated in lower energy states, one may expect the upper limit to be a fair approximation of the spectroscopic factor. Table 1 includes orbital occupancies calculated using the USD interaction as well as spectroscopic factors where available. The measured spectroscopic factor for the $0.885-\mathrm{MeV}$ and $0.765-\mathrm{MeV}$ states agree well with the SDPF-M upper limits for the $1 / 2^{+}$and $3 / 2^{-}$strengths, respectively. This evidence, along with results from the momentum distribution and the level structure, supports a tentative assignment of $1 / 2^{+}$for the $0.885 \mathrm{MeV}$ state and $3 / 2^{-}$for the $0.765 \mathrm{MeV}$ state.

In summary, individual states in ${ }^{25} \mathrm{Ne}$ and ${ }^{27} \mathrm{Ne}$ have been populated in oneneutron knockout reactions. For ${ }^{25} \mathrm{Ne}$, the level scheme, spin assignments, and spectroscopic strengths are in agreement with USD shell-model calculations. Spin and parity $1 / 2^{+}$could be unambiguously assigned to the ground state of ${ }^{25} \mathrm{Ne}$. USD and SDPF-M shell model predictions agree for this nucleus, indicating that intruder states do not play a role for the low-lying level scheme of this $N=15$ nucleus. With just two neutrons more, ${ }^{27} \mathrm{Ne}$ is predicted to have low-lying negative-parity intruder states by the SPDF-M Monte-Carlo shell-model calculations, while the USD shell model yields a $1 / 2^{+}$state as the only bound, excited state. Two excited states have been observed in the present experiment. The $\ell$-values deduced from the longitudinal momentum distributions of the ${ }^{27} \mathrm{Ne}$ knockout residues and the spectroscopic factors obtained in comparison to eikonal reaction theory support the identification of 
the $0.765 \mathrm{MeV}$ and $0.885 \mathrm{MeV}$ excited states as the $3 / 2^{-}$and $1 / 2^{+}$levels, respectively. This result confirms the importance of intruder states in the ground state configuration of ${ }^{28} \mathrm{Ne}$ and the predictive power of the SDPF-M Monte Carlo Shell Model calculations in this transitional region.

\section{Acknowledgments}

This work is supported by the United States National Science Foundation under contracts PHY-0110253 and PHY-0244453, by the UK Engineering and Physical Sciences Research Council (EPSRC) under Grant No. EP/D003628, by a Grant-in-Aid for Specially Promoted Research (13002001) and a Grant-inAid for Young Scientists (14740176) from the MEXT and by the RIKEN-CNS collaboration project on large-scale nuclear structure calculation.

\section{References}

[1] T. Otsuka et al., Phys. Rev. Lett. 87 (2001) 082502.

[2] E. Warburton and B.A. Brown, Phys. Rev. C 46 (1992) 923.

[3] A. Ozawa et al., Phys. Rev. Lett. 84 (2000) 5493.

[4] M. Stanoiu et al., Phys. Rev. C 69 (2004) 034312.

[5] A. Obertelli et al., Phys. Rev. C 71 (2005) 024304.

[6] Y. Utsuno et al., Phys. Rev. C 60 (1999) 054315.

[7] H. Sakurai et al., Phys. Lett. B 448 (1999) 180.

[8] C. Thibault et al., Phys. Rev. C 12 (1975) 644.

[9] D.J. Vieira et al., Phys. Rev. Lett. 57 (1986) 3253.

[10] C. Détraz et al., Phys. Rev. C 19 (1979) 164.

[11] T. Motobayashi et al., Phys. Lett. B 346 (1995) 9.

[12] J.A. Church et al., Phys. Rev. C 72 (2005) 054320.

[13] E. Warburton et al., Phys. Rev. C 41 (1990) 1147.

[14] M. Honma et al., Phys. Rev. Lett. 75 (1995) 1284.

[15] Y. Utsuno et al., Phys. Rev. C 64 (2001) 011301(R).

[16] B.A. Brown, Prog. Part. Nucl. Phys. 47 (2001) 517. 
[17] Y. Utsuno et al., Phys. Rev. C 70 (2004) 044307.

[18] V. Tripathi et al., Phys. Rev. Lett. 94 (2005) 162501.

[19] M. Belleguic et al., Phys. Rev. C 72 (2005) 054316.

[20] A. Obertelli et al., Phys. Lett. B 633 (2006) 33.

[21] P.G. Hansen and J.A. Tostevin, Annu. Rev. Nucl. Part. Sci. 53 (2003) 219.

[22] F. Marti et al., In Proceedings of the $16^{\text {th }}$ Conference on Cyclotrons and Their Applications, edited by F. Marti (American Institute of Physics, East Lansing, Michigan, 2001) Vol. 600, p. 64.

[23] D.J. Morrissey et al., Nucl. Instrum. Methods Phys. Res. B 204 (2003) 90.

[24] D. Bazin et al., Nucl. Instrum. Methods Phys. Res. B 204 (2003) 629.

[25] W.F. Mueller et al., Nucl. Instrum. Methods Phys. Res. A 466 (2001) 492.

[26] A. Gade et al., Phys. Rev. C 71 (2005) 051301(R).

[27] A.T. Reed et al., Phys. Rev. C 60 (1999) 024311.

[28] S.W. Padgett et al., Phys. Rev. C 72 (2005) 064330.

[29] A. Gade et al., Phys. Rev. C 69 (2004) 034311.

[30] C.A. Bertulani, P.G. Hansen, Phys. Rev. C 70 (2004) 034609.

[31] J.A. Tostevin et al., Nucl. Phys. A 682 (2001) 320c.

[32] J.A. Tostevin et al., J. Phys. G 25 (1999) 735.

[33] W. Catford et al., Eur. Phys. J. A 25, s01 (2005) 245.

[34] B.A. Brown, Phys. Rev. C 58 (1998) 220.

[35] A. Gade et al., Phys. Rev. Lett. 93 (2004) 042501. 


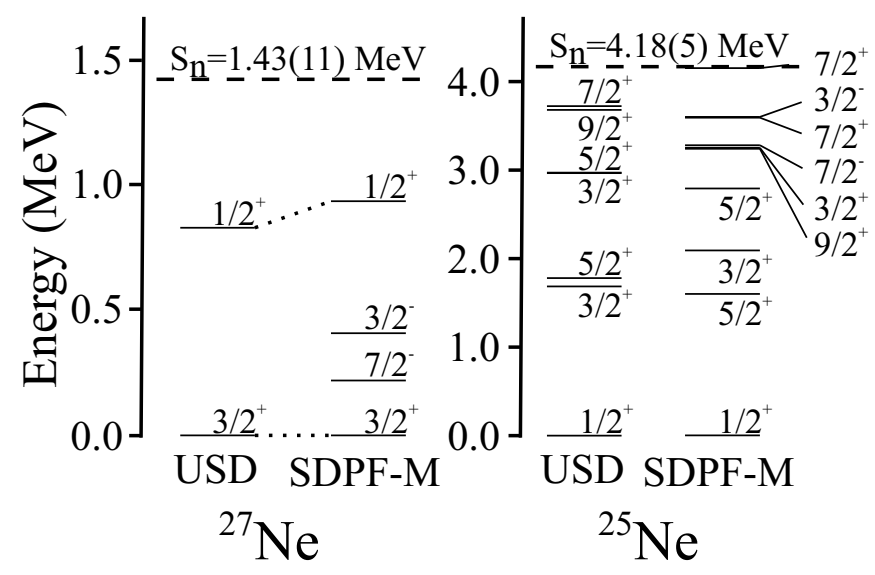

Fig. 1. All bound states predicted for ${ }^{25,27} \mathrm{Ne}$ by USD and SDPF-M calculations.

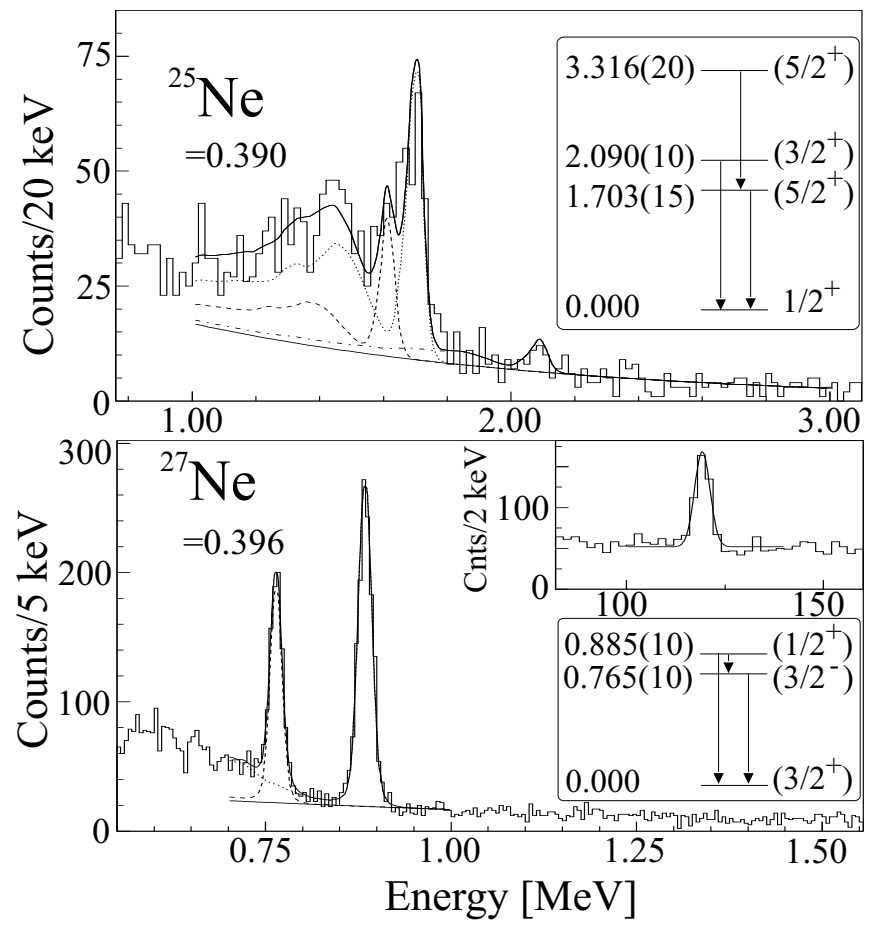

Fig. 2. Coincident Doppler-reconstructed $\gamma$-ray energy spectra from single-neutron removal from ${ }^{26,28} \mathrm{Ne}$. The dashed lines represent simulated response functions of SeGA while the solid lines show results of the fit to the spectrum. 


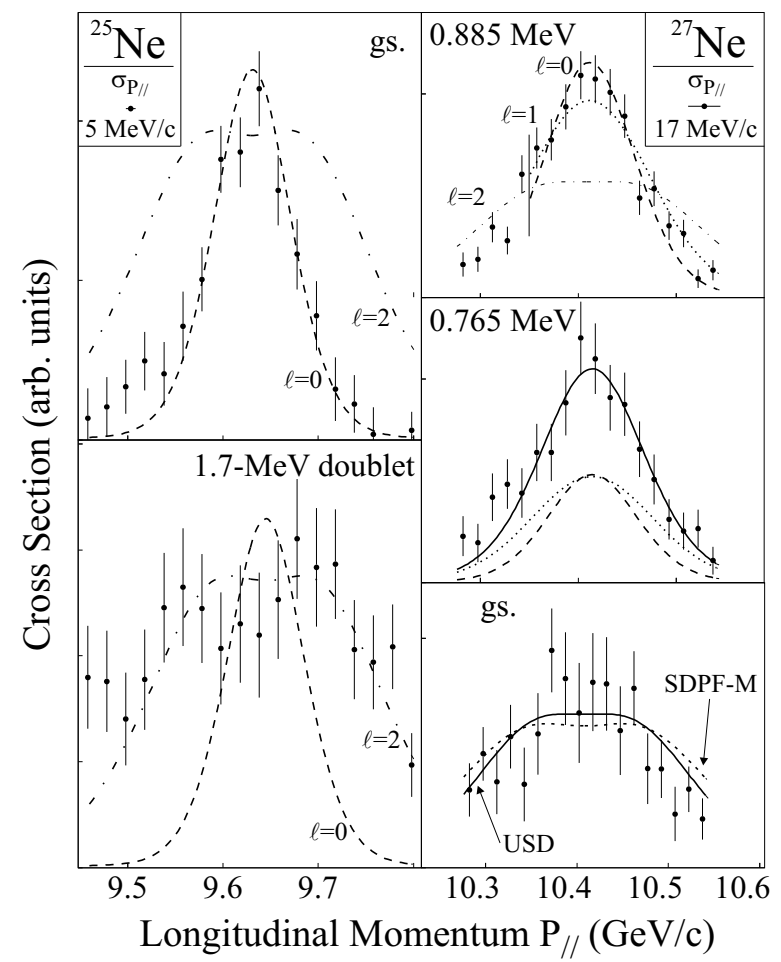

Fig. 3. Longitudinal momentum distributions of the ${ }^{25,27}$ Ne knockout residues. The $1-\sigma$ momentum resolutions for ${ }^{25} \mathrm{Ne}$ and ${ }^{27} \mathrm{Ne}$ are shown in the upper panel for each isotope. Dashed, dotted, and dash-dotted lines in the ${ }^{25} \mathrm{Ne}$ panels and the upper ${ }^{27} \mathrm{Ne}$ panel correspond to calculated distributions assuming $\ell=0, \ell=1$, and $\ell=2$. Lines in the lower two ${ }^{27} \mathrm{Ne}$ panels represent combinations of $\ell$-values discussed in the text. Calculated distributions are convoluted with the known momentum resolution. 


\begin{tabular}{|c|c|c|c|c|c|c|c|c|}
\hline $\begin{array}{l}\mathrm{E}_{\text {level }} \\
(\mathrm{MeV})\end{array}$ & $\ell$ & $\begin{array}{r}\sigma_{e x p} \\
(\mathrm{mb})\end{array}$ & $\begin{array}{l}\sigma_{s p} \\
(\mathrm{mb})\end{array}$ & $\begin{array}{l}\mathrm{C}^{2} \mathrm{~S} \\
\exp \end{array}$ & $\mathrm{J}^{\pi}$ & $\begin{array}{l}\mathrm{C}^{2} \mathrm{~S} \\
\mathrm{USD}\end{array}$ & $\begin{array}{c}\langle\mathrm{n}\rangle \\
\mathrm{USD}\end{array}$ & $\begin{array}{c}\langle\mathrm{n}\rangle \\
\text { SDPF-M }\end{array}$ \\
\hline \multicolumn{9}{|c|}{${ }^{9} \mathrm{Be}\left({ }^{26} \mathrm{Ne},{ }^{25} \mathrm{Ne}\right) \mathrm{X}$} \\
\hline 0.000 & 0 & $42(4)$ & 36.6 & $1.2(1)$ & $\frac{1}{2}^{+}$ & 1.25 & 1.61 & 1.74 \\
\hline 1.703 & 2 & $25(2)$ & 21.1 & $1.2(1)$ & \multirow{2}{*}{$\frac{5}{2}^{+}$} & 2.17 & \multirow{2}{*}{5.67} & \multirow{2}{*}{5.68} \\
\hline 3.316 & 2 & $22(3)$ & 19.0 & $1.1(2)$ & & 1.70 & & \\
\hline \multirow[t]{4}{*}{2.090} & $2^{*}$ & $9(2)$ & 20.0 & $0.5(1)$ & $\frac{3}{2}^{+}$ & 0.38 & 0.72 & 0.48 \\
\hline & & & & & $\frac{7}{2}^{-}$ & - & - & 0.08 \\
\hline & & & & & $\frac{3}{2}^{-}$ & - & - & 0.02 \\
\hline & \multicolumn{2}{|c|}{$\sigma_{i n c}=98(5)$} & & & & $\Sigma=$ & 8 & 8 \\
\hline \multicolumn{9}{|c|}{${ }^{9} \mathrm{Be}\left({ }^{28} \mathrm{Ne},{ }^{27} \mathrm{Ne}\right) \mathrm{X}$} \\
\hline 0.000 & & $21(2)$ & & & $\frac{3}{2}^{+}$ & 1.75 & 2.18 & 1.18 \\
\hline 0.765 & $(0,1)$ & $10(1)$ & 32.7 & $0.32(4)$ & $\frac{3}{2}^{-}$ & - & - & 0.24 \\
\hline \multirow[t]{4}{*}{0.885} & $(0,1)$ & $35(2)$ & 32.7 & $1.07(7)$ & $\frac{1}{2}^{+}$ & 1.50 & 1.93 & 1.87 \\
\hline & & & & & $\frac{7}{2}^{-}$ & - & - & 0.89 \\
\hline & & & & & $\frac{5}{2}^{+}$ & - & 5.88 & 5.83 \\
\hline & \multicolumn{2}{|c|}{$\sigma_{i n c}=66(3)$} & & & & $\Sigma=$ & 10 & 10 \\
\hline
\end{tabular}

*Deduced from Refs. [28,33]

Table 1

Results for the ${ }^{9} \mathrm{Be}\left({ }^{26} \mathrm{Ne},{ }^{25} \mathrm{Ne}\right) \mathrm{X}$ and ${ }^{9} \mathrm{Be}\left({ }^{28} \mathrm{Ne},{ }^{27} \mathrm{Ne}\right) \mathrm{X}$ reactions. The deduced level energies as well as the measured $\ell$-value and partial cross sections are given. Singleparticle cross sections $\sigma_{s p}$ are calculated in a three-body eikonal model [31,32]. Since the $\ell$ value for the ${ }^{27} \mathrm{Ne}$ excited states can only be constrained, the calculated singleparticle cross sections are taken as the average of the $\ell=0$ and $\ell=1$ results, the values of which differ by less than $1 \mathrm{mb}$. Uncertainties for $\sigma_{\exp }$ and $\mathrm{C}^{2} \mathrm{~S}_{\exp }$ include a $7 \%$ systematic uncertainty for ${ }^{26} \mathrm{Ne}$ and $6 \%$ for ${ }^{28} \mathrm{Ne}$. We also give the predicted spins and parities along with the corresponding spectroscopic factors $\mathrm{C}^{2} \mathrm{~S}$ for the bound states predicted from USD shell model calculations and single-particle occupancies $\langle\mathrm{n}\rangle$ from both USD and SDPF-M calculations. 